\title{
Application of Mixture Design Response Surface Methodology for Combination Chemotherapy in PC-3 Human Prostate Cancer Cells $\mathbf{s}$
}

\author{
Richard Oblad, Hayden Doughty, John Lawson, Merrill Christensen, and Jason Kenealey
}

Department of Nutrition, Dietetics, and Food Science (R.O., H.D., M.C., J.K.) and Statistics (J.L.), Brigham Young University, Provo, Utah

Received December 30, 2017; accepted June 7, 2018

\begin{abstract}
Combining chemotherapeutics to treat malignant tumors has been shown to be effective in preventing drug resistance, tumor recurrence, and reducing tumor size. We modeled combination drug therapy in $\mathrm{PC}-3$ human prostate cancer cells using mixture design response surface methodology (MDRSM), a statistical technique designed to optimize compositions that we applied in a novel manner to design combinations of chemotherapeutics. Conventional chemotherapeutics (mitoxantrone, cabazitaxel, and docetaxel) and natural bioactive compounds (resveratrol, piperlongumine, and flavopiridol) were used in 12 different combinations containing three drugs at varying concentrations. Cell viability
\end{abstract}

and cell cycle data were collected and used to plot response surfaces in MDRSM that identified the most effective concentrations of each drug in combination. MDRSM allows for extrapolation of data from three or more compounds in variable ratio combinations, unlike the Chou-Talalay method. MDRSM combinations were compared with combination index data from the Chou-Talalay method and were found to coincide. We propose MDRSM as an effective tool in devising combination treatments that can improve treatment effectiveness and increase treatment personalization, because MDRSM measures effectiveness rather than synergism, potentiation, or antagonism.

\section{Introduction}

Drug resistance is one of the greatest problems facing chemotherapy, is believed to be the cause of over $90 \%$ of failed treatments of metastatic cancer patients in 2005 , and continues to be a major problem to this day (Longley and Johnston, 2005; Kachalaki et al., 2016). Personalized and targeted therapies, such as immunotherapy, have shown great potential in combating cancer but are still affected by chemoresistance (Bozic et al., 2013). Prostate cancer frequently becomes resistant to commonly used treatments (Theyer et al., 1993; Tilley et al., 1995; Li et al., 2001; Liang et al., 2010) and is the second most commonly diagnosed cancer in men in the United States (Siegel et al., 2018). Of the diagnosed prostate cancers, $10 \%$ are metastatic (Jemal et al., 2011). Metastatic prostate cancer is incurable and highly fatal (Kim et al., 2003, 2004; Hegeman et al., 2004; Patel et al., 2004; Pinthus et al., 2004; Gundem et al., 2015). PC-3 human prostate cancer cells, a cell line derived from metastatic prostate cancer, have been shown to be chemoresistant to

This work was funded by the Brigham Young University Life Science Startup Grant.

The authors declare no conflict of interest.

https://doi.org/10.1124/mol.117.111450.

S This article has supplemental material available at molpharm. aspetjournals.org. some common chemotherapeutics (Theyer et al., 1993; Tilley et al., 1995; Li et al., 2001). One way to combat chemoresistance is combination drug therapy. Combination therapy uses a combination of drugs with different mechanistic actions. It has also been theorized that multimechanistic drugs may be helpful in preventing the development of resistance (Zimmermann et al., 2007). Many naturally occurring compounds such as resveratrol (RES), flavopiridol (FLAV), and piperlongumine (PIP) are multimechanistic compounds that have been identified as putative chemotherapeutics (Pan et al., 2012). Treatment with RES has been shown to cause increases in cytosolic calcium (Ma et al., 2007; Peterson et al., 2016), bind to various proteins to induce intrinsic apoptosis (Locatelli et al., 2005; Calamini et al., 2010; Hsieh et al., 2014), and cause production of ROS (Miki et al., 2012). FLAV inhibits cyclin dependent kinases to disrupt the cell cycle and causes production of ROS (Gupta et al., 2010; Shao et al., 2016), whereas PIP has been shown to cause ROS production and inhibit signal transducer and activator of transcription 3 and nuclear factor kappa-light-chain-enhancer of activated B cells (Kong et al., 2008; Adams et al., 2012; Ginzburg et al., 2014; Gong et al., 2014; Bharadwaj et al., 2015). Cabazitaxel (CAB), docetaxel (DOC), and mitoxantrone (MIT) are all conventional chemotherapeutics that are used to treat prostate cancer (Tannock et al., 2004; de Bono et al.,

ABBREVIATIONS: CAB, cabazitaxel; CSC, cancer stem cell; DMSO, dimethylsulfoxide; DOC, docetaxel; FLAV, flavopiridol; MDRSM, mixture design response surface methodology; MIT, mitoxantrone; PIP, piperlongumine; RES, resveratrol; ROS, reactive oxygen species; RSM, response surface methodology. 
2010; Paller and Antonarakis, 2011). CAB and DOC are both tubulin inhibitors (Jordan and Wilson, 2004; Galsky et al., 2010). However, resistance develops to DOC but not to CAB in some forms of prostate cancer, likely because $\mathrm{CAB}$ has a lower binding affinity to the drug efflux pump P-glycoprotein (Galsky et al., 2010; Paller and Antonarakis, 2011; OPREALAGER et al., 2013). Mitoxantrone is a DNA intercalating agent and topoisomerase II inhibitor that prevents DNA replication (Ehninger et al., 1990). DOC, CAB, and MIT are all used in treating advanced prostate cancer and have often been used in combination therapies (Petrylak et al., 2004; de Bono et al., 2010).

The most commonly used statistical model to define and test chemotherapeutic drug combinations is the Chou-Talalay method for measuring drug synergism (two-way complement between drugs) and antagonism (two-way interference between drugs) (Chou, 2010). However, the Chou-Talalay method is limited by only: 1) directly analyzing drug combinations of two drugs, 2) focusing on constant ratio combinations, and 3) measuring synergism that is not directly correlated with effectiveness. The Chou-Talalay method uses the 1:1 mixtures of the two drugs being tested at concentrations that are multiples of the $\mathrm{IC}_{50}(0.50 \times, 1.0 \times, 2.0 \times$, and $\left.4.0 \times \mathrm{IC}_{50}\right)$ and requires collection of data of each drug alone (Chou and Talalay, 1983, 1984). By using Chou-Talalay data, one infers how three drug interactions may work, but never directly measure how synergistic the drugs are together. Chou recognized this limitation when he stated that combinations do not act as two different drugs but act as a third drug altogether (Chou, 2010). The advantage of measuring nonconstant ratio combinations is that nonconstant ratios provide a broader range of mixtures that enable the discovery of novel drug interactions. The Chou-Talalay method can be used to test variable ratio combinations, but it requires a significant amount of data collection. Lastly, to find the most effective chemotherapeutic mix, one is not necessarily interested in the drugs with the strongest synergism, but the ultimate goal is to identify the combination that is most effective at reducing the tumor.

Response surface methodologies (RSM) are a compilation of mathematical and statistical models that create a 3-dimensional map based on interpolating defined data points. A number of different RSMs have been used in cancer research. For example, the Box-Behnken design was used to optimize the incorporation of chemotherapeutics into nanoparticles (Gupta et al., 2016). Additionally, fractional factorial design was used to maximize extraction of putative chemotherapeutics from plants (Lu et al., 2008), and PlackettBurman was used to optimize the growth media for tumor cells to enable the discovery of a potential cancer vaccine (Zhao et al., 2017). To a lesser extent, RSM has also been used to optimize combination chemotherapy. For example, a multifactorial design was used to identify the most effective chemotherapeutic combination in prostate cancer (Jia et al., 2017). Given how widely used RSM is in engineering and other fields, it is unclear why it has been underutilized in chemotherapy.

Mixture design response surface methodology (MDRSM) is a type of RSM that can test combinations with two or more compounds and interpolate effective mixtures from nonconstant ratio combinations. MDRSM can be constrained by desired parameters as designated by the researcher, such as making the model define a combination that has between $20 \%$ and $80 \%$ of one drug (Dejaegher and Heyden, 2011). MDRSM has been used in many other fields to create the most effective or desirable product, such as soil composition for a landfill, the most desirable mixture of ingredients for any given food product, and to develop pharmaceutical formulations (Cornell, 2011). MDRSM can be used to develop maximally effective drug combinations for treating cancer, because it is not limited to measuring only synergism or antagonism but takes into account any potentiation and indicates the most effective combination. Although a mixture of two or three drugs is most common in drug combination studies, MDRSM can be used to optimize combinations of two or more compounds. The effects of such mixtures on outcomes such as cell viability or cell cycle inhibitions are experimentally determined and normalized to a control. The derived data for percent viability or percent inhibition are then used to construct the response surface. The surface predicts the outcome of any combination of the tested compounds, including the unique blend that would minimize or maximize the desired response. In this study, we compare MDRSM to the Chou-Talalay model and test 12 different combinations of three drugs using six different chemotherapeutic agents. We demonstrate that MDRSM can be used to determine the efficacy of three drug mixtures in nonconstant ratios.

\section{Materials and Methods}

\section{Cell Lines}

PC-3 human prostate cancer cells were obtained from ATCC (Rockville, MD) and were cultured in F12-K medium containing $10 \%$ FBS and $1 \%$ antibiotic (penicillin/streptomycin). Cells were grown in an incubator at $37^{\circ} \mathrm{C}$ and $5 \% \mathrm{CO}_{2}$. Cells from passage numbers $3-17$ were used.

\section{Reagents}

Cabazitaxel was obtained from APExBIO (Houston, TX) and dissolved in dimethylsulfoxide (DMSO) to make a $40 \mu \mathrm{M}$ stock solution. Docetaxel, flavopiridol, mitoxantrone hydrochloride, and piperlongumine were obtained from Sigma Aldrich (St. Louis, MO) and were dissolved in DMSO to make a 45, 160, $400 \mu \mathrm{M}$, and $8 \mathrm{mM}$ stock solution, respectively. Trans-resveratrol was obtained from Cayman Chemical (Ann Arbor, MI) and dissolved in DMSO to make a $75 \mathrm{mM}$ stock solution. Alamarblue was obtained from Remel (Lenexa, KS). Propidium iodide was obtained from Sigma Aldrich and dissolved in phosphate-buffered saline for a stock concentration of $50 \mu \mathrm{g} / \mathrm{ml}$.

\section{Cell Viability}

Cells were grown near confluency, trypsinized, and 10,000 cells were seeded on 96 -well plates. Cells were allowed to adhere to the plate for 24 hours before treatment with the vehicle control (DMSO $0.1 \%$ ) or 1 of 10 drug combinations and allowed to incubate for 48 hours $\left(37^{\circ} \mathrm{C}\right.$ and $\left.5 \% \mathrm{CO}_{2}\right)$. DMSO only control was set as $100 \%$ viability. Each data point was normalized to the DMSO control and is presented as a percent reduction in cell viability so that the strongest reduction in cell viability would give us a maximum on the surface. Each combination treatment also had its own control where treatment medium, without cells, was analyzed with Alamarblue to eliminate background fluorescence and interactions between the drugs and the Alamarblue reagent. After 48 hours, the cells were treated with $10 \mu \mathrm{l}$ of Alamarblue (1:10 ratio of Alamarblue to media), and incubated for 6 hours $\left(37^{\circ} \mathrm{C}\right.$ and $\left.5 \% \mathrm{CO}_{2}\right)$. The fluorescence was then analyzed on a 


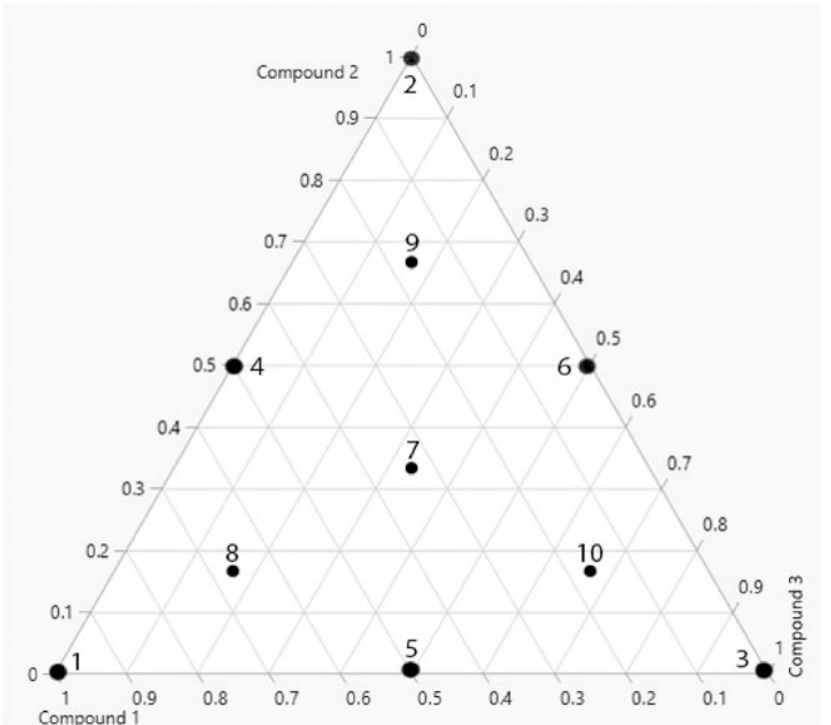

Fig. 1. JMP ternary plot with combination points. Ternary plot of a threecompound combination containing the 10 points used to define a surface. Concentrations for each point are read by following the left diagonal lines for compound 1, horizontal lines for compound 2, and right diagonal lines for compound 3 .

BMG LABTECH FLUOstar OPTIMA plate reader (BMG LABTECH Inc., Cary, NC) using the $544 \mathrm{~nm}$ excitation filter and the $612 \mathrm{~nm}$ emission filter. All treatments were done with three biologic replicates and in triplicate within each biologic replicate.

\section{IC $_{50}$ Values}

The cell viability method above was used to collect data for varying concentrations above and below the $\mathrm{IC}_{50}$ as shown in Fig. 2. GraphPad Prism 7 (Graphpad Software, San Diego, CA) was used to fit a variable slope non-linear regression model to the data that had been normalized to a control (DMSO). After the model identified the $\mathrm{IC}_{50}$ we further refined the $\mathrm{IC}_{50}$ by testing several drug concentrations near the calculated $\mathrm{IC}_{50}$ to establish a drug concentration that reliably reduced cell viability by $50 \%$. The data for the additional experiments and the statistics for the models can be found in Supplemental Tables 1-7.

Flow Cytometry. Cells were grown near confluency, trypsinized, and seeded on 12-well plates at 150,000 cells per well. Cells were allowed to adhere to the plate for 24 hours before treatment with the specified 10 combinations or the vehicle control (DMSO $0.2 \%$ ) for 48 hours (incubated at $37^{\circ}$ and $5 \% \mathrm{CO}_{2}$ ). After 48 hours, the treatment medium was removed, and the cells were washed twice with phosphate-buffered saline before being trypsinized and harvested. To each aliquot of cells, $500 \mu \mathrm{l}$ of $70 \% \mathrm{EtOH}$ was added, and samples were stored at $4^{\circ} \mathrm{C}$. When analyzed, samples were stained with propidium iodide, and 40,000 counts per sample were collected on a BD Accuri C6 Flow cytometer (BD Bioscience, San Jose, CA). Gates were drawn for the DMSO control using BD Accuri C6 Software, and the same gates were used for data collection from each sample for data collection. Percentages of cells in $G_{0}, G_{1}, S$, and $\mathrm{G}_{2} / \mathrm{M}$ phases were calculated and normalized to the DMSO control. All samples were done in triplicate.

\section{Chou-Talalay}

Cell viability assays (Alamarblue) for $0.25 \times, 0.50 \times, 1 \times, 2 \times$, and $4 \times$ $\mathrm{IC}_{50}$ for each dual combination of the six drugs was performed and normalized to a $0.8 \%$ DMSO control. The data were input into the program CompuSyn (ComboSyn Inc., Paramus, NJ), as well as the concentration ratios, to find the combination index for each
TABLE 1

Ternary plot coordinate system represented as percentages-100\% can be artificially set to any desired concentration

\begin{tabular}{lcccc}
\hline & Ternary Point & Compound 1 & $\begin{array}{c}\text { Compound } 2 \\
\%\end{array}$ & Compound 3 \\
\hline 1 & 1 & 100 & 0 & 0 \\
2 & 2 & 0 & 100 & 0 \\
3 & 3 & 0 & 0 & 100 \\
4 & 4 & 50 & 50 & 0 \\
5 & 5 & 50 & 0 & 50 \\
6 & 6 & 0 & 50 & 50 \\
7 & 7 & 33.33 & 33.33 & 33.33 \\
8 & 8 & 66.67 & 16.67 & 16.67 \\
9 & 9 & 16.67 & 66.67 & 16.67 \\
10 & 10 & 16.67 & 16.67 & 66.67 \\
\hline
\end{tabular}

combination to determine synergism or antagonism. All data were collected and analyzed as directed by the Chou-Talalay method (Chou and Talalay, 1983).

\section{Mixture Design and Model}

The statistical software JMP 12 (SAS Institute, Cary, NC) was used to create and analyze the response surfaces. A simplex lattice $(3,2)$ mixture design, augmented by a center point and three axial points, was used (Fig. 1) (Cornell, 2011). Each mixture combination was replicated three times resulting in a design with 30 total experiments. After each experiment was conducted, two responses, cell viability and cell cycle analysis, were obtained and recorded. Next, the method of least squares was used with the resulting data to estimate the coefficients in the quadratic mixture model used to define the response surface (Cornell, 2011). The quadratic mixture model is based on a Taylor series expansion with a second-degree polynomial (eq. 1).

$$
\eta=\beta_{a} X_{a}+\beta_{b} X_{b}+\beta_{c} X_{c}+\beta_{a b} X_{a} X_{b}+\beta_{a c} X_{a} X_{c}+\beta_{b c} X_{b} X_{c}
$$

where $\eta$ is the predicted response, $\beta$ is the slope of the response, and $X$ is the concentration of the compounds that are indicated by the subscripts $a, b$, and $c$. The predicted maximum for each combination was identified using the JMP profiler (that allows visualization of cross sections of the predicted response surface as one or two mixture components are varied at a time). The resulting maximum can also be identified on a contour plot of the fitted quadratic model over the simplex design region (for combinations of three compounds only). The lack of fit F-test was performed to check the adequacy of the quadratic model. Statistical significance of the lack of fit was determined by a $P$ value $\leq 0.10$.

\section{Response Surfaces}

The coordinate system for the simplex is present on each ternary plot. In Fig. 1, the axes for compound 1 are negative sloping lines, the axes for compound 2 are parallel lines (zero slope), and the axes for compound 3 are positive sloping lines. For example, point 10 on Fig. 1 represents $16.67 \%$ compound 1 (following negative sloping lines to the bottom of the plot), $16.67 \%$ compound 2 (following the parallel lines to the left side of the plot), and $66.67 \%$ compound 3 (following the positive sloping lines to the right side of the plot). Three combinations are in the corners of the ternary plot (points 1, 2, and 3 of Fig. 1) and represent the use of only the single compound specified in that corner. Three combinations (points 4, 5, and 6 of Fig. 1) are in the middle of the axis between two corners and represent a 50-50 combination of the two compounds named at those corners. The other four points $(7,8,9$, and 10 of Fig. 1) are located in the middle of the ternary plot, and represent mixtures containing various concentrations of all three compounds. The 10 points create an even spread over the plot that produces a strong surface for differentiating mixtures (Dejaegher and Heyden, 2011). Adding more compounds to the mixture design increases the amount of combinations that will need to be empirically tested to 

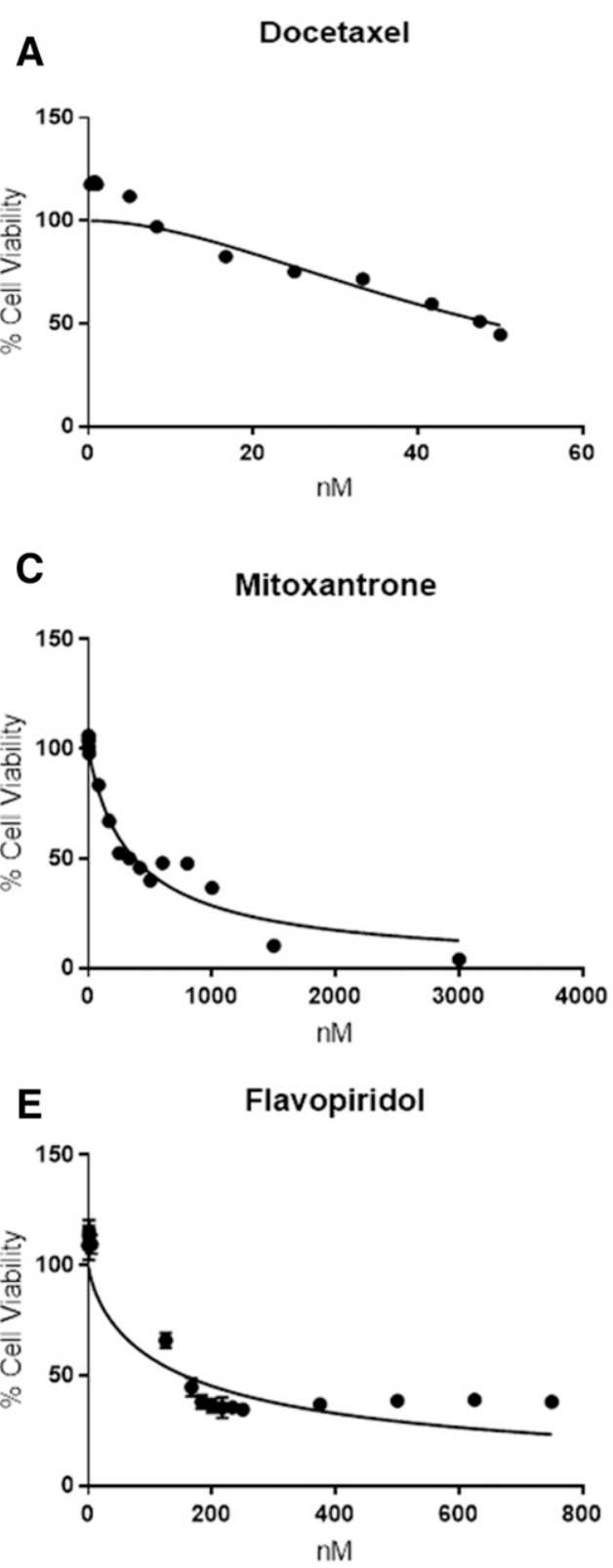

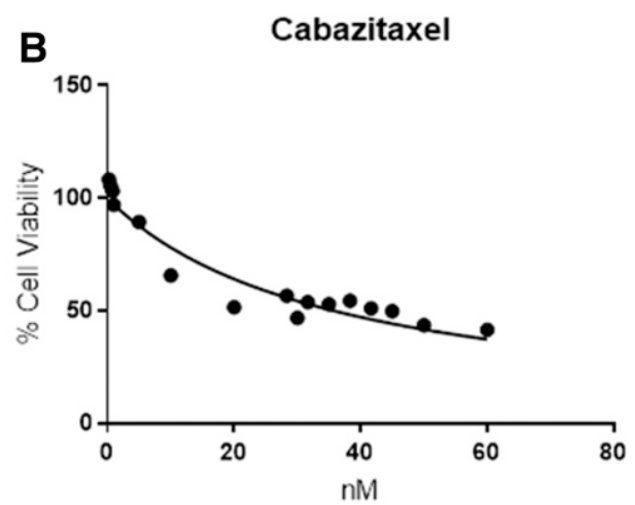

D

Resveratrol

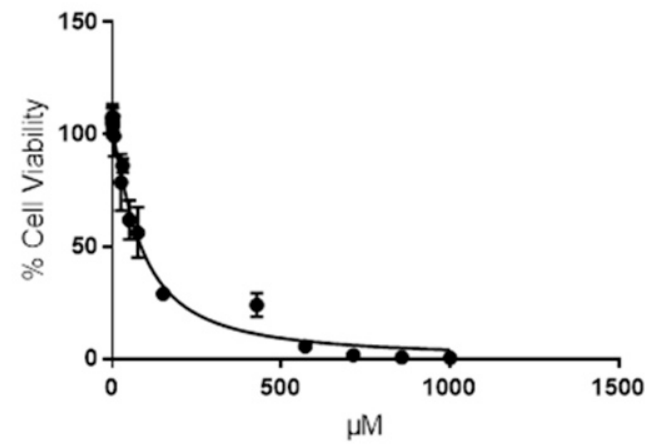

$\mathbf{F}$

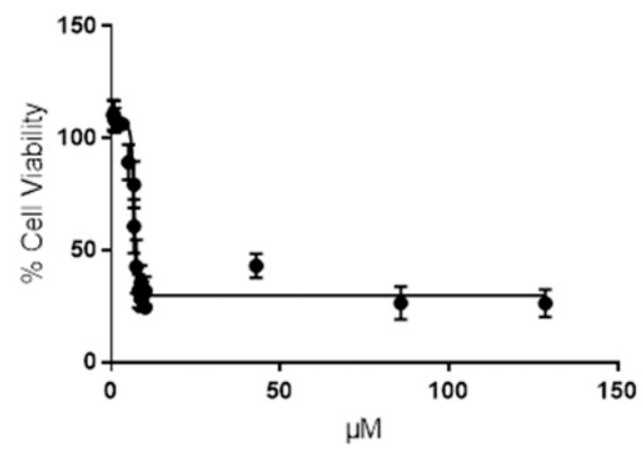

Fig. 2. $\mathrm{IC}_{50}$ graphs for chemotherapeutics in PC-3 human prostate cancer cells. (A) DOC, $45 \mathrm{nM}$; (B) CAB, $40 \mathrm{nM}$; (C) MIT, $400 \mathrm{nM}$; (D) RES, $75 \mu \mathrm{M}$; (E) FLAV, $160 \mathrm{nM}$; (F) PIP, $7 \mu$ M. $n=3$.

define the response surface. For example, optimization of a mixture of 10 compounds would require testing of at least 66 different combinations. Here we focused on combinations of three compounds, which require only 10 points. The coordinate system produced on the simplex represents a percentage of a selected maximum concentration for each compound (Table 1). The maximum for each compound can be set to any concentration. For example, $100 \%$ of compound 1 could be set to the maximum tolerable dose, whereas the maximum of compound 2 is set to $3 \times$ the $\mathrm{IC}_{50}$ and compound 3 to a therapeutic dose. We set the maximum concentration to the $\mathrm{IC}_{50}$ for each compound (Fig. 2), as determined by Alamarblue cell viability assay in PC-3 human prostate cancer cells. The $\mathrm{IC}_{50}$ was used as the maximum specifically to have direct comparisons with the $0.5 \times \mathrm{IC}_{50}$ combination index from the Chou-Talalay method (correlates with points 4,5 , and 6 of the MDRSM model). We used Alamarblue to measure relative cell viability of the 10 points and set our DMSO control as 100\% viability. We subtracted the normalized cell viability from 100 to express the data as a percent reduction in cell viability so that the strongest reduction in cell viability would give us a maximum on the surface. The maximum, or ideal combination, can easily be identified by the model. The predicted points defined by the model are given $95 \%$ confidence intervals and allow for statistical conclusions to be made about that point by comparing the confidence intervals.

\section{Results}

Drug Combination Modeling Using MDRSM. We tested 12 different combinations. We limited each combination to three of the six total compounds (Fig. 3). Each data set is represented in a ternary plot. The ternary plots can be read like topographical maps. The red area indicates the area with the largest decrease in tumor cell viability. The dark red area is the next most effective chemotherapeutic mixtures, with gray, dark blue, and blue indicating the areas with 
A

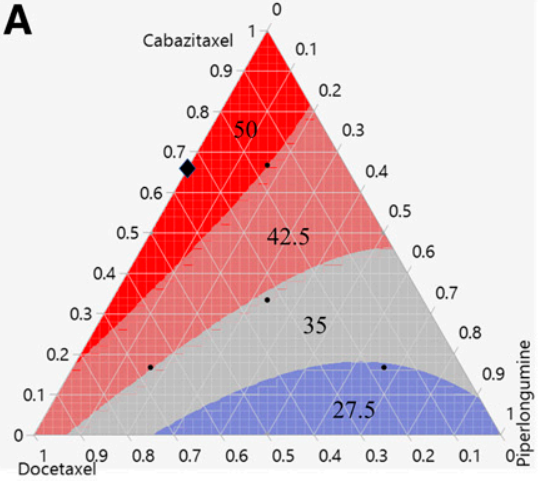

D

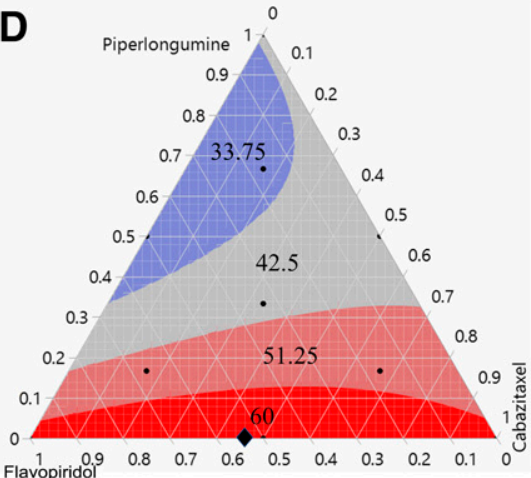

G

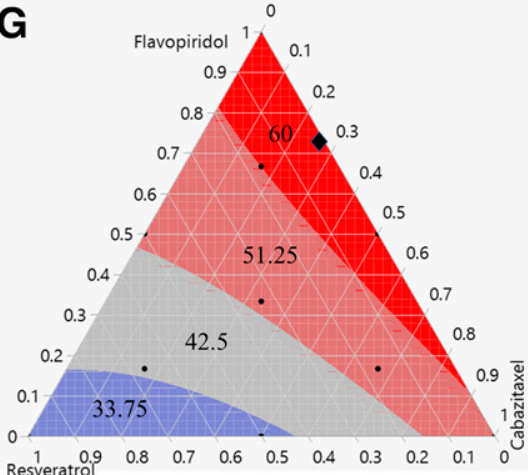

$\mathbf{J}$

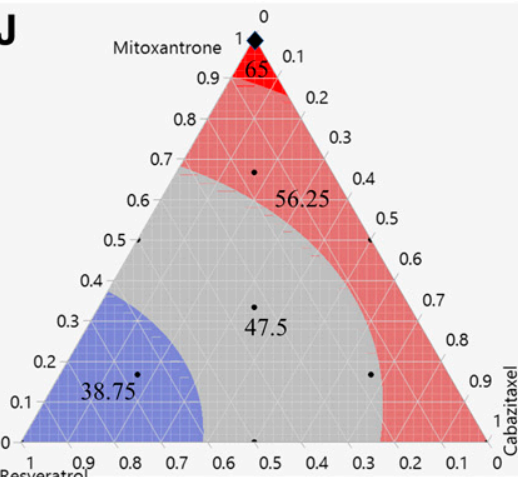

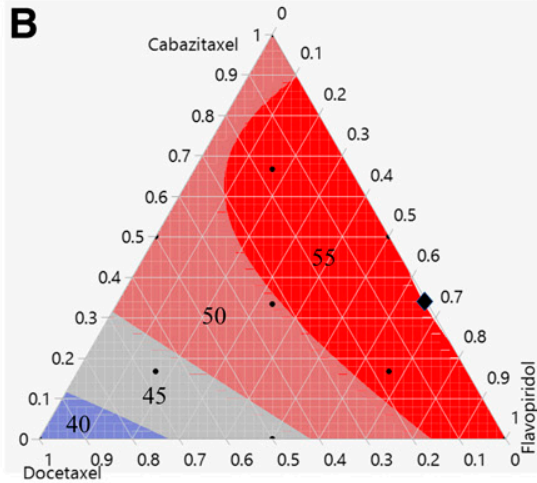
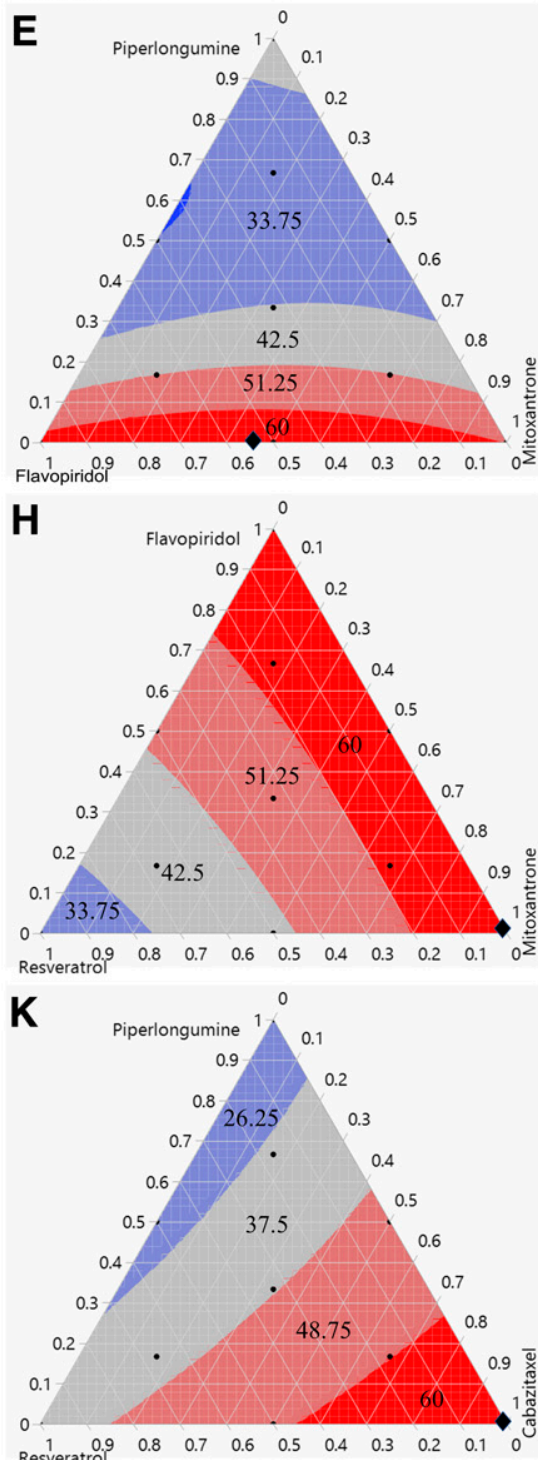

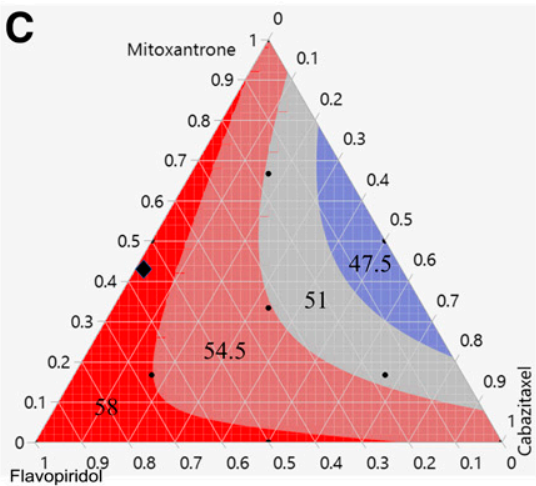

$\mathbf{F}$
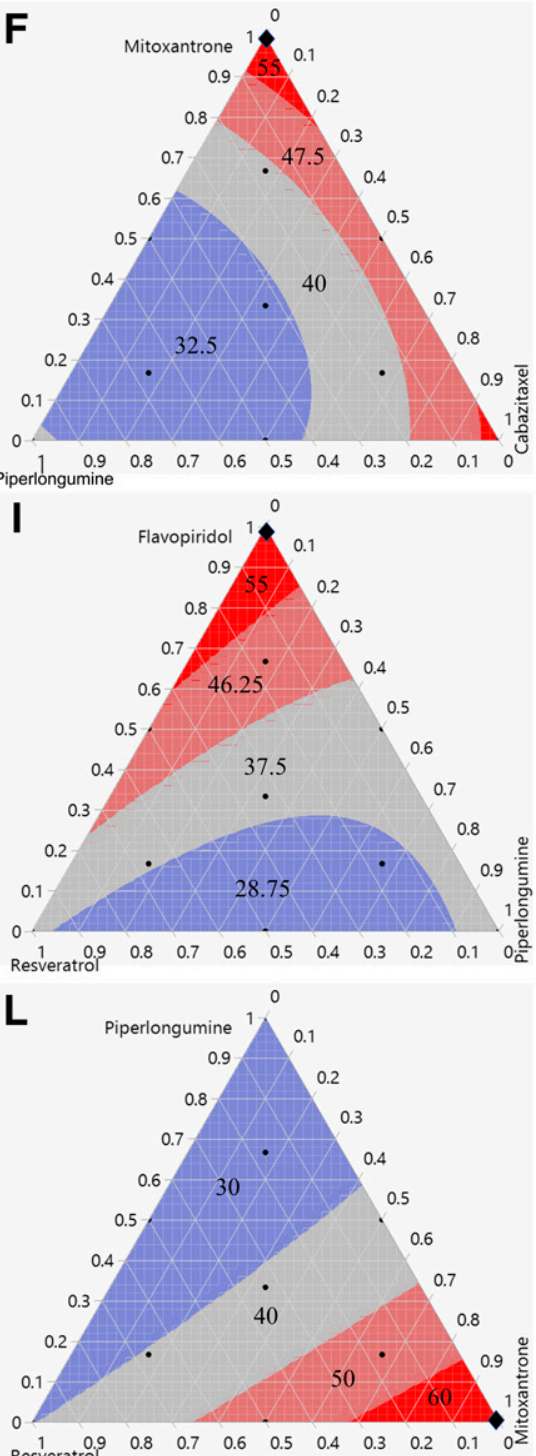

Fig. 3. Response surfaces of 12 different mixtures. Bright red areas represent the most effective combinations at reducing cell viability. Deep red, gray, and blue are listed in descending order of effectiveness. The numbers within each contour represent the maximum prediction found within that contour, meaning that all predictions within that contour are less than or equal to the number found within but greater than the numbers found in the lower contours. The maximum point on the surface is indicated by a diamond. The mixtures for each ternary plot are: (A) DOC, CAB, PIP; (B) DOC, CAB, FLAV; (C) FLAV, MIT, CAB; (D) FLAV, PIP, CAB; (E) FLAV, PIP, MIT; (F) PIP, MIT, CAB; (G) RES, FLAV, CAB; (H) RES, FLAV, MIT; (I) RES, FLAV, PIP; (J) RES, MIT, CAB; (K) RES, PIP, CAB; (L) RES, PIP, MIT. All data were obtained by Alamarblue cell viability assay with three biologic replicates in triplicate.

successively lower decrease in tumor cell viability. Ten combinations were made with every possible three-component arrangements of five compounds (DOC not included), and two combinations were formulated with $\mathrm{DOC}$ and $\mathrm{CAB}$ together. Combinations containing DOC always contained $\mathrm{CAB}$ to determine if MDRSM could differentiate between possible 
additive effects produced by drugs with similar mechanisms; however, we found a synergistic interaction between them. $\mathrm{CAB}$ was used in the rest of the combinations because it was effective at a slightly lower concentration than DOC.

We found that CAB is more effective than DOC when combined with PIP, and the surface favors a combination of $\mathrm{CAB}$ and DOC while excluding PIP from the mixture (Fig. 3A). The model reveals the maximum reduction in cell viability to be $33 \%$ DOC, $67 \% \mathrm{CAB}$, and $0 \%$ PIP (Table 2 ), which causes a $45 \%$ decrease in viability. When $\mathrm{DOC}$ and $\mathrm{CAB}$ are combined with FLAV (Fig. 3B), the surface favors a combination of FLAV $(67.36 \%)$ and CAB $(32.64 \%)$ and causes a $52 \%$ decrease in cell viability, indicating that antitumor effect of $\mathrm{CAB}$ is stronger than DOC when combined with FLAV. This does not match the Chou-Talalay data that will be discussed later. The data support the ability of MDRSM to identify the most effective combinations, even when those drugs have similar mechanisms.

Each compound in the combination has an equal role in defining the surface, even if that compound is not included in the most effective combination. This phenomenon can cause the most effective combination to change between surfaces. For example, the most effective combination for DOC-CABFLAV (Fig. 3B) is $0 \%$ DOC, 33\% CAB, and 67\% FLAV, but the most effective combination for FLAV-PIP-CAB (Fig. 3D) is $54 \%$ FLAV, $0 \%$ PIP, and $46 \%$ CAB. The change of $13 \%$ in concentrations is caused by the presence of PIP in the model, and therefore such results should be confirmed with another combination model or by reducing the MDRSM model to look only at combinations between the two compounds. The $13 \%$ decrease is not a significant difference when the confidence intervals are compared, but with more precise measurements, or a mixture using a higher concentration than the $\mathrm{IC}_{50}$, the difference may be significant.

Our three drug mixtures also demonstrated that PIP, an effective agent against cancer when used in isolation, decreases the effectiveness of the other compounds when in combination (Fig. 3, A, D-F, I, K, and L). PIP does not appear to be effective in combination with any of the other compounds that we tested, which the model shows by removing it from any combination that it has been used in. This limited effectiveness of PIP in combination with other compounds may be in part due to its steep Hill slope. The $\mathrm{IC}_{50}$ for $\mathrm{PIP}$ is $7 \mu \mathrm{M}$, and the next highest concentration used is $4.7 \mu \mathrm{M}$, which only reduces tumor cell viability by $15 \%$. Subsequent experiments could be done redefining the mixture ratios to remain within the effective dose of PIP. The effects of PIP are not only due to its steep Hill slope, however, as RES has a similar effect to PIP, but does not have the steep Hill slope. RES causes the effective area between RES (0\%)-FLAV (0\%)-MIT (100\%) (Fig. 3H) to change entirely compared with FLAV (56\%)-PIP (0\%)-MIT (44\%) (Fig. 3E). PIP does not appear to disrupt the effectiveness of combinations like RES. RES disrupts the effectiveness of combinations, causing the surface to favor the use of only one compound, except between FLAV-CAB (Fig. 3G). The model reveals that $0.28 \%$ RES slightly increases the effectiveness of (potentiates) FLAV and CAB; however, the concentration of resveratrol is not a significant amount and becomes $0 \%$ (Table 2). The presence of RES in the model shifts the concentration higher for FLAV and lower for CAB compared with Fig. 3A (33\% CAB and 67\% FLAV) to $73 \%$ FLAV and 27\% CAB (Table 2). The handling of RES and PIP in these combinations identifies the ability of MDRSM to find the most effective combinations in the presence of antagonism. The antagonistic actions identified by RES and PIP coincide with antagonism measured by the Chou-Talalay method (Fig. 4).

Of the 12 combinations performed, only one combination (FLAV-MIT-CAB Fig. 3C) called for a mixture of all three compounds (Table 2). The combinations favor higher percentages of FLAV (55\%) and MIT (44\%) while only having a minute amount of $\mathrm{CAB}(1 \%)$ Fig. 3C). However, the predicted maximum viability decreases of $56 \%$ and $95 \%$ confidence interval of $44 \%-69 \%$ suggests that there is not a significant difference in reduced viability from that slight addition of $\mathrm{CAB}$ (Table 2). As stated previously, this may be significant if higher concentrations were used or if a more precise response was measured.

The Chou-Talalay Method Identifies Synergism and Confirms Some MDRSM Mixtures. MDRSM identifies the optimal concentration, but the Chou-Talalay method differentiates between synergism, additive, and antagonistic effects. The combination index curve produced by the ChouTalalay equation cannot be extrapolated to include combinations with nonconstant ratios without collecting significantly more data. As per our design, the equal mixture of $0.5 \times \mathrm{IC}_{50}$ of

TABLE 2

Optimal concentration and percentages for maximizing a reduction in cell viability

\begin{tabular}{|c|c|c|c|c|c|c|c|c|c|c|}
\hline & \multirow[b]{2}{*}{ Combination } & \multicolumn{2}{|c|}{ Compound 1} & \multicolumn{2}{|c|}{ Compound 2} & \multicolumn{2}{|c|}{ Compound 3} & \multirow{2}{*}{$\begin{array}{l}\text { Predicted } \\
\text { Decrease } \\
\text { in \% Cell } \\
\text { Viability }\end{array}$} & \multirow{2}{*}{$\begin{array}{c}95 \% \\
\text { Confidence } \\
\text { Interval }\end{array}$} & \multirow{2}{*}{$\begin{array}{l}\text { Lack of Fit } \\
\text { Prob }>F^{*}\end{array}$} \\
\hline & & $\begin{array}{c}\text { Percentage } \\
\text { of } \mathrm{IC}_{50}\end{array}$ & Concentration & $\begin{array}{c}\text { Percentage } \\
\text { of } \mathrm{IC}_{50}\end{array}$ & Concentration & $\begin{array}{l}\text { Percentage } \\
\text { of } \mathrm{IC}_{50}\end{array}$ & Concentration & & & \\
\hline 1 & DOC, CAB, PIP & 33 & $15 \mathrm{nM}$ & 67 & $27 \mathrm{nM}$ & 0 & $0 \mu \mathrm{M}$ & 45 & 39,52 & 0.7069 \\
\hline 2 & DOC, CAB, FLAV & 0 & $0 \mathrm{nM}$ & 33 & $13 \mathrm{nM}$ & 67 & $108 \mathrm{nM}$ & 52 & 45,59 & 0.5903 \\
\hline 3 & FLAV, MIT, CAB & 55 & $89 \mathrm{nM}$ & 44 & $176 \mathrm{nM}$ & 1 & $0.4 \mathrm{nM}$ & 56 & 44,69 & 0.2957 \\
\hline 4 & FLAV, PIP, CAB & 54 & $86 \mathrm{nM}$ & 0 & $0 \mu \mathrm{M}$ & 46 & $18 \mathrm{nM}$ & 61 & 46,76 & 0.9568 \\
\hline 5 & FLAV, PIP, MIT & 56 & $90 \mathrm{nM}$ & 0 & $0 \mu \mathrm{M}$ & 44 & $175 \mathrm{nM}$ & 58 & 40,76 & 0.9961 \\
\hline 6 & PIP, MIT, CAB & 0 & $0 \mu \mathrm{M}$ & 100 & $400 \mathrm{nM}$ & 0 & $0 \mathrm{nM}$ & 55 & 35,75 & 0.5780 \\
\hline 7 & RES, FLAV, CAB & 0 & $0 \mu \mathrm{M}$ & 73 & $117 \mathrm{nM}$ & 27 & $11 \mathrm{nM}$ & 56 & 48,63 & 0.8182 \\
\hline 8 & RES, FLAV, MIT & 0 & $0 \mu \mathrm{M}$ & 0 & $0 \mathrm{nM}$ & 100 & $400 \mathrm{nM}$ & 61 & 46,76 & 0.9841 \\
\hline 9 & RES, FLAV, PIP & 0 & $0 \mu \mathrm{M}$ & 100 & $160 \mathrm{nM}$ & 0 & $0 \mu \mathrm{M}$ & 50 & 36,65 & 0.9657 \\
\hline 10 & RES, MIT, CAB & 0 & $0 \mu \mathrm{M}$ & 100 & $400 \mathrm{nM}$ & 0 & $0 \mathrm{nM}$ & 58 & 44,72 & 0.5081 \\
\hline 11 & RES, PIP, CAB & 0 & $0 \mu \mathrm{M}$ & 0 & $0 \mu \mathrm{M}$ & 100 & $40 \mathrm{nM}$ & 54 & 43,65 & 0.4811 \\
\hline 12 & RES, PIP, MIT & 0 & $0 \mu \mathrm{M}$ & 0 & $0 \mu \mathrm{M}$ & 100 & $400 \mathrm{nM}$ & 58 & 44,73 & 0.9852 \\
\hline
\end{tabular}

*Values less than or equal to 0.10 indicate that the model does not fit the data. 
the Chou-Talalay data are included in the MDRSM data as a 50-50 mixture (points 4, 5, and 6 of Table 1) between two compounds. Interestingly, there is synergism between DOC$\mathrm{CAB}$ at $0.5 \times \mathrm{IC}_{50}$ (Fig. 4), which is close to what is identified as the most effective dose in the MDRSM seen in Fig. 3A (33\% DOC, 67\% CAB, 0\% PIP). The MDRSM model accounts for that point and still identifies the ideal combination as $33 \%$ DOC and $67 \%$ CAB (Table 2), although this may be influenced by PIP. In the mixture of DOC-CAB-FLAV (Fig. 3B) there is stronger synergism between DOC-FLAV (0.63441) than CABFLAV (0.79421) or DOC-CAB (0.73087) (Fig. 4). However, MDRSM places the ideal combination between CAB-FLAV, because it is measuring the effectiveness of the combination and not the synergism. Perhaps this is due to the ability of $\mathrm{PC}-3$ cells to become resistant to DOC more readily than $\mathrm{CAB}$, which makes the CAB-FLAV mixture more effective.

The Chou-Talalay model identified synergism between CAB and FLAV (Fig. 4) that correlates with most effective dose identified using MDRSM data. MDRSM places the optimal concentration between CAB and FLAV in Fig. 3, B, D, and G. The concentrations vary between mixtures, but this is due to the change in the surface caused by the third compound. The optimal concentration identified by MDRSM appears to correlate strongly with synergistic actions identified by the Chou-Talalay method, and we conclude that the most effective mixture identified by MDRSM is strongly affected by synergism, but not in every circumstance. MDRSM provides an advantage over the Chou-Talalay model because it identifies the most effective combination irrespective of the mechanism.

Cell Cycle Data Are a Valid Input for MDRSM. To further show the robustness of MDRSM, we performed cell cycle analysis for the 10-point mixture of CAB-FLAV-MIT, because all three drugs have a different effect on the cell cycle. CAB inhibits microtubule formation, FLAV inhibits cyclin dependent kinases, and MIT inhibits topoisomerase II and prevents DNA unwinding (Ehninger et al., 1990; Galsky et al., 2010; Gupta et al., 2010). We treated the samples with the 10 combinations of three compounds described above, with the maximum concentration being $80 \mathrm{nM}$ CAB, $320 \mathrm{nM}$ FLAV, and $800 \mathrm{nM}$ MIT to see pronounced effects on the cell cycle. After 48 hours of treatment, we harvested the cells, stained them with propidium iodide, and used a flow cytometer to analyze the cell cycle phase for cells treated with the 10 mixtures. These data were then normalized to the DMSO control and used to produce the surfaces in Fig. 5. The combination that gives the largest decrease of cells in the $\mathrm{G}_{1}$ phase is a combination that includes CAB (Fig. 5A), and the model shows $100 \%$ CAB (Table 3). However, the lack of fit is significant $(P$ value $=0.0087)$, meaning the model does not fit the data properly or the combination defined by this model is not significant. Maximizing the number of cells in the S phase requires a mixture of $\mathrm{MIT}$ and $\mathrm{CAB}$ (Fig. $5 \mathrm{~B}$ ), which is $32 \%$ CAB and 68\% MIT (Table 3). This is perhaps due to the inability of cells to divide, caused by the microtubule inhibitor $\mathrm{CAB}$, and the DNA damage, as well as halting of DNA replication by MIT. The most effective dose at trapping cells in the $\mathrm{G}_{2} / \mathrm{M}$ phase is $100 \% \mathrm{CAB}$ because of the inability of the cells to form microtubules and divide (Fig. 5C). Maximizing cells in the sub $\mathrm{G}_{1}$ phase, or causing the most damage to DNA, requires a combination of MIT and FLAV (Fig. 5D), and the model shows the ideal combination is $48 \% \mathrm{MIT}$ and $52 \% \mathrm{FLAV}$ (Table 3). Comparisons between the MDRSM model produced

\begin{tabular}{|l|c|c|c|c|c|}
\hline & $\mathbf{0 . 2 5}$ X Ic50 & $\mathbf{0 . 5}$ X Ic50 & $\mathbf{1 ~ X ~ I c 5 0 ~}$ & $\mathbf{2 ~ X ~ I c 5 0 ~}$ & $\mathbf{4 ~ X ~ I c 5 0 ~}$ \\
\hline Cab, Flav & 1.94 & 0.79 & 0.67 & 1.00 & 2.74 \\
\hline Cab, Mit & 4.53 & 2.85 & 1.61 & 1.08 & 1.33 \\
\hline Cab, Pip & 16.70 & 2.04 & 4.08 & 0.82 & 1.85 \\
\hline Cab, Res & 4.05 & 2.68 & 1.61 & 2.71 & 1.86 \\
\hline Doc, Flav & 0.79 & 0.63 & 0.55 & 0.93 & 2.16 \\
\hline Doc, Pip & 1.44 & 1.15 & 1.20 & 0.93 & 2.05 \\
\hline Doc, Cab & 1.06 & 0.73 & 1.32 & 1.62 & 3.39 \\
\hline Flav, Res & 3.39 & 1.30 & 0.71 & 1.33 & 1.94 \\
\hline Mit, Flav & 1.46 & 1.05 & 0.60 & 1.24 & 2.15 \\
\hline Mit, Pip & 2.73 & 3.81 & 3.01 & 1.12 & 2.36 \\
\hline Mit, Res & 1.23 & 2.01 & 1.88 & 2.25 & 2.14 \\
\hline Pip, Flav & 70299.50 & 2.01 & 0.94 & 1.30 & 2.71 \\
\hline Pip, Res & 1.81 & 1.49 & 1.18 & 0.99 & 2.41 \\
\hline
\end{tabular}

Fig. 4. Chou-Talalay combination index (CI) chart for all combinations. Red represents synergistic CIs, gray represents additive CIs, and blue represents antagonistic CIs. CIs were all obtained by Alamarblue cell viability assay with three biologic replicates in triplicate.

by cell viability (Fig. 3C), and the models produced here cannot be made because the same concentrations were not used. However, comparisons between cell viability and cell cycle phase, when the same concentrations are used, may help to determine the mechanism by which the drugs are reducing cell viability, whether it be cell death, halting of the cell cycle, or a mixture of both. It is interesting to note that the ratio between drugs in the cell viability treatment and that of the sub $\mathrm{G}_{1}$ phase are similar even though the concentrations are different.

\section{Discussion}

In this work, we reviewed the basic uses and application of MDRSM in combination chemotherapy. We show several models of three-drug combinations and the limitations and advantages of MDRSM. We demonstrate that cell viability and cell cycle assays are valid inputs for creating a surface. We demonstrate beneficial combinations for treating PC-3 human prostate cancer cells between standard chemotherapeutics and FLAV, a bioactive compound derived from a natural compound. We show that RES and PIP can interfere with standard chemotherapeutics. However, we speculate that RES may potentiate CAB-FLAV. We also show that the optimal combination defined by MDRSM is affected by the response that is chosen to define the surface. MDRSM, in companion with the Chou-Talalay method, can be used to identify strong combination treatments that can increase the effectiveness of chemotherapy.

MDRSM has the potential to bring a new perspective to developing drug combinations. MDRSM can be used to measure the effectiveness of drug mixtures targeting specific aspects of cancer such as those defined in the hallmarks of cancer (Hanahan and Weinberg, 2011). Furthermore, by collecting multiple responses for one combination, such as the effects of the combinations on healthy tissue as well as cancerous tissue, the models for each response can be merged into a new model known as the desirability function. The desirability function can be used to maximize the effect on cancerous tissue, while minimizing the effects on healthy tissue. Developing such a treatment would be a major step forward in improving the quality of life for cancer patients. 

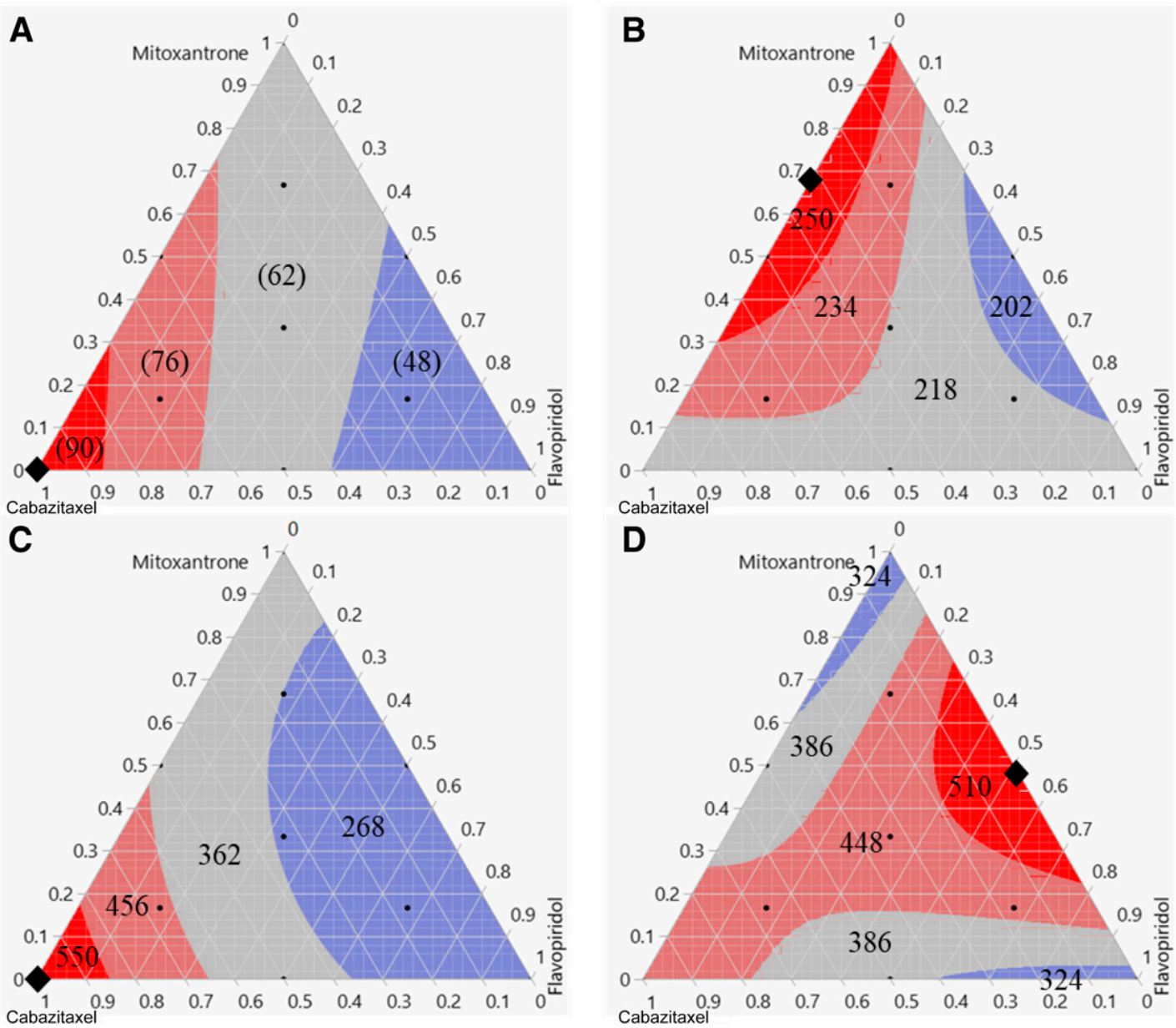

Fig. 5. Response surfaces produced by cell cycle analysis. Bright red areas represent the most effective combinations. Deep red, gray, and blue are listed in descending order of effectiveness. The numbers within each contour represent the maximum prediction found within that contour, meaning that all predictions within that contour are less than or equal to the number found within but greater than the numbers found in the lower contours. The maximum point on the surface is indicated by a diamond. (A) Surface for relative decrease in $\mathrm{G}_{1}$ phase populations. (B) Surface for maximizing $\mathrm{S}$ phase populations. (C) Surface for maximizing $\mathrm{G}_{2} / \mathrm{M}$ phase populations. (D) Surface for maximizing sub $\mathrm{G}_{1}$ populations. All data were obtained from flow cytometry of propidium iodide stained cells. All samples were analyzed in triplicate.

MDRSM could also be used to develop treatments based on the prominent theory of tumor heterogeneity (Singh and Settleman, 2010), which is the existence of frequently chemoresistant tumorigenic cancer stem cells (CSC) that produce a genetically diverse tumor. This theory cites the existence of resistant CSCs to be the cause of chemoresistance and tumor recurrence (Marusyk and Polyak, 2010; Housman et al., 2014). MDRSM could model a combination of common chemotherapeutics along with a targeted treatment of CSCs that could lead to maximum tumor reduction and minimal risk of tumor recurrence.
Although we collected 10 data points for each simplex lattice design, more data points could have been added to the design. Adding more points to a design can allow for fitting a higher order polynomial model (such as cubic or quartic) to the data; however, this is not guaranteed to make the fitted model more reliable. When fitting an empirical model, a higher order term may just model the random experimental noise. Normally in response surface methods, a quadratic model based on the two term Taylor series expansion is sufficient to identify interactions, and the combination of compounds predicted to result in the maximum response. However, MDRSM can be used in an

TABLE 3

Optimum combinations for minimizing cell population in the $\mathrm{G}_{1}$ phase and maximizing cell populations in the $\mathrm{S}, \mathrm{G}_{2} / \mathrm{M}$, and sub $G_{1}$ phases produced by cell cycle analysis

\begin{tabular}{lccccccc}
\hline & Phase & Cabazitaxel & Mitoxantrone & Flavopiridol & $\begin{array}{c}\text { \% Change in } \\
\text { Population }\end{array}$ & $\begin{array}{c}\text { 95\% Confidence } \\
\text { Interval }\end{array}$ & $\begin{array}{c}\text { Lack of Fit } \\
\text { Prob }>\text { F }\end{array}$ \\
\hline 1 & $\mathrm{G}_{1}$ phase & $100 \%, 80 \mathrm{nM}$ & $0 \%, 0 \mathrm{nM}$ & $0 \%, 0 \mathrm{nM}$ & $(86)$ & $(78),(95)$ & $0.0087^{*}$ \\
2 & S phase & $32 \%, 26 \mathrm{nM}$ & $68 \%, 543 \mathrm{nM}$ & $0 \%, 0 \mathrm{nM}$ & 238 & 208,268 & 0.1722 \\
3 & $\mathrm{G}_{2} / \mathrm{M}$ phase & $100 \%, 80 \mathrm{nM}$ & $0 \%, 0 \mathrm{nM}$ & $0 \%, 0 \mathrm{nM}$ & 537 & 469,606 & 0.5320 \\
4 & $\mathrm{Sub} \mathrm{G}_{1}$ phase & $0 \%, 0 \mathrm{nM}$ & $48 \%, 387 \mathrm{nM}$ & $52 \%, 165 \mathrm{nM}$ & 499 & 312,687 & 0.7216 \\
\hline
\end{tabular}

*Values less than or equal to 0.10 indicate that the model does not fit the data. 
iterative process to refine further the optimal mixtures. This can be accomplished by performing subsequent $\operatorname{MDRSM}(\mathrm{s})$, but this time defining the points more narrowly within the region identified in the preceding MDRSM as the optimal mixture.

MDRSM is a powerful tool for measuring combination chemotherapy, but the data can easily be overinterpreted. For example, a response for MDRSM could be as simple as a cell viability experiment in a cell model, but the ideal combination given by the surface would only be ideal for reducing cell viability. Response surfaces produced by an apoptotic assay or cell cycle analysis may have similar results as the cell viability surface, but the differences between ideal combinations will be specific for the response. One can compare multiple responses of the same combination of drugs in MDRSM by merging the models to form a desirability function as mentioned above. Choosing the response for MDRSM should be specific to the cell, or animal model, and should be reflective of a desired effect for combating the specific type of cancer being researched. A potential limitation of MDRSM is the choice of maximum concentrations. Preliminary data from a Chou-Talalay combination index analysis may be helpful in choosing a maximum concentration.

We propose MDRSM as an effective tool for modeling and defining drug combinations in cell, animal, and clinical models. MDRSM can also be a tool in combating chemoresistance by using a combination of drugs at their maximal effectiveness for decreasing tumor size, while minimizing the ability of the remaining tumor cells to become resistant. MDRSM has the ability to develop combinations using two or more drugs and provides a novel and improved way of measuring the effectiveness of multiple compounds. MDRSM in combination with the Chou-Talalay method can cover a broad area of drug combinations, providing a multiplicity of data while being both cost and time effective.

\section{Authorship Contributions}

Participated in research design: Oblad, Christensen, Kenealey.

Conducted experiments: Oblad, Doughty.

Contributed analytical tools: Lawson, Christensen.

Performed data analysis: Oblad, Lawson.

Wrote or contributed to the writing of the manuscript: Oblad, Doughty, Lawson, Christensen, Kenealey.

\section{References}

Adams DJ, Dai M, Pellegrino G, Wagner BK, Stern AM, Shamji AF, and Schreiber SL (2012) Synthesis, cellular evaluation, and mechanism of action of piperlongumine analogs. Proc Natl Acad Sci USA 109:15115-15120.

Bharadwaj U, Eckols TK, Kolosov M, Kasembeli MM, Adam A, Torres D, Zhang X, Dobrolecki LE, Wei W, Lewis MT, et al. (2015) Drug-repositioning screening identified piperlongumine as a direct STAT3 inhibitor with potent activity against breast cancer. Oncogene 34:1341-1353.

Bozic I, Reiter JG, Allen B, Antal T, Chatterjee K, Shah P, Moon YS, Yaqubie A, Kelly N, Le DT, et al. (2013) Evolutionary dynamics of cancer in response to targeted combination therapy. eLife 2:e00747.

Calamini B, Ratia K, Malkowski MG, Cuendet M, Pezzuto JM, Santarsiero BD, and Mesecar $\mathrm{AD}$ (2010) Pleiotropic mechanisms facilitated by resveratrol and its metabolites. Biochem J 429:273-282.

Chou TC (2010) Drug combination studies and their synergy quantification using the Chou-Talalay method. Cancer Res 70:440-446.

Chou T-C and Talalay P (1983) Analysis of combined drug effects: a new look at a very old problem. Trends Pharmacol Sci 4:450-454

Chou T-C and Talalay P (1984) Quantitative analysis of dose-effect relationships: the combined effects of multiple drugs or enzyme inhibitors. Adv Enzyme Regul 22: $27-55$

Cornell JA (2011) Experiments With Mixtures: Designs, Models, and the Analysis of Mixture Data, John Wiley \& Sons, New York.

de Bono JS, Oudard S, Ozguroglu M, Hansen S, Machiels J-P, Kocak I, Gravis G, Bodrogi I, Mackenzie MJ, Shen L, et al.; TROPIC Investigators (2010) Prednisone plus cabazitaxel or mitoxantrone for metastatic castration-resistant prostate cancer progressing after docetaxel treatment: a randomised open-label trial. Lancet 376:1147-1154

Dejaegher B and Heyden YV (2011) Experimental designs and their recent advances in set-up, data interpretation, and analytical applications. J Pharm Biomed Anal 56:141-158.

Ehninger G, Schuler U, Proksch B, Zeller K-P, and Blanz J (1990) Pharmacokinetics and metabolism of mitoxantrone. A review. Clin Pharmacokinet 18:365-380.

Galsky MD, Dritselis A, Kirkpatrick P, and Oh WK (2010) Cabazitaxel. Nat Rev Drug Discov 9:677-678.

Ginzburg S, Golovine KV, Makhov PB, Uzzo RG, Kutikov A, and Kolenko VM (2014) Piperlongumine inhibits NF- $\mathrm{KB}$ activity and attenuates aggressive growth characteristics of prostate cancer cells. Prostate 74:177-186.

Gong LH, Chen XX, Wang H, Jiang QW, Pan SS, Qiu JG, Mei XL, Xue YQ, Qin WM, Zheng FY, et al. (2014) Piperlongumine induces apoptosis and synergizes with cisplatin or paclitaxel in human ovarian cancer cells. Oxid Med Cell Longev 2014: 906804.

Gundem G, Van Loo P, Kremeyer B, Alexandrov LB, Tubio JMC, Papaemmanuil E, Brewer DS, Kallio HML, Högnäs G, Annala M, et al.; ICGC Prostate Group (2015) The evolutionary history of lethal metastatic prostate cancer. Nature 520:353-357.

Gupta B, Poudel BK, Pathak S, Tak JW, Lee HH, Jeong J-H, Choi H-G, Yong CS, and Kim JO (2016) Effects of formulation variables on the particle size and drug encapsulation of imatinib-loaded solid lipid nanoparticles. AAPS PharmSciTech 17:652-662.

Gupta SC, Kim JH, Prasad S, and Aggarwal BB (2010) Regulation of survival, proliferation, invasion, angiogenesis, and metastasis of tumor cells through modulation of inflammatory pathways by nutraceuticals. Cancer Metastasis Rev 29: 405-434.

Hanahan D and Weinberg RA (2011) Hallmarks of cancer: the next generation. Cell 144:646-674.

Hegeman RB, Liu G, Wilding G, and McNeel DG (2004) Newer therapies in advanced prostate cancer. Clin Prostate Cancer 3:150-156.

Housman G, Byler S, Heerboth S, Lapinska K, Longacre M, Snyder N, and Sarkar S (2014) Drug resistance in cancer: an overview. Cancers (Basel) 6:1769-1792.

Hsieh TC, Lin C-Y, Bennett DJ, Wu E, and Wu JM (2014) Biochemical and cellular evidence demonstrating AKT-1 as a binding partner for resveratrol targeting protein NQO2. PLoS One 9:e101070.

Jemal A, Bray F, Center MM, Ferlay J, Ward E, and Forman D (2011) Global cancer statistics. CA Cancer J Clin 61:69-90.

Jia X, Li Y, Sharma A, Li Y, Xie G, Wang G, Jiang J, Cheng Y, and Ding X (2017) Application of sequential factorial design and orthogonal array composite design (OACD) to study combination of 5 prostate cancer drugs. Comput Biol Chem 67: $234-243$.

Jordan MA and Wilson L (2004) Microtubules as a target for anticancer drugs. Nat Rev Cancer 4:253-265.

Kachalaki S, Ebrahimi M, Mohamed Khosroshahi L, Mohammadinejad S, and Baradaran B (2016) Cancer chemoresistance; biochemical and molecular aspects: a brief overview. Eur J Pharm Sci 89:20-30.

Kim SJ, Johnson M, Koterba K, Herynk MH, Uehara H, and Gallick GE (2003) Reduced c-Met expression by an adenovirus expressing a c-Met ribozyme inhibits tumorigenic growth and lymph node metastases of PC3-LN4 prostate tumor cells in an orthotopic nude mouse model. Clin Cancer Res 9:5161-5170.

Kim SJ, Uehara H, Yazici S, Langley RR, He J, Tsan R, Fan D, Killion JJ, and Fidler IJ (2004) Simultaneous blockade of platelet-derived growth factor-receptor and epidermal growth factor-receptor signaling and systemic administration of paclitaxel as therapy for human prostate cancer metastasis in bone of nude mice. Cancer Res 64:4201-4208.

Kong EH, Kim YJ, Cho HJ, Yu SN, Kim KY, Chang JH, and Ahn SC (2008) Piplartine induces caspase-mediated apoptosis in PC-3 human prostate cancer cells. Oncol Rep 20:785-792.

Li X, Marani M, Mannucci R, Kinsey B, Andriani F, Nicoletti I, Denner L, and Marcelli M (2001) Overexpression of BCL-X(L) underlies the molecular basis for resistance to staurosporine-induced apoptosis in PC-3 cells. Cancer Res 61: $1699-1706$

Liang X-J, Meng H, Wang Y, He H, Meng J, Lu J, Wang PC, Zhao Y, Gao X, Sun B, et al. (2010) Metallofullerene nanoparticles circumvent tumor resistance to cisplatin by reactivating endocytosis. Proc Natl Acad Sci USA 107:7449-7454.

Locatelli GA, Savio M, Forti L, Shevelev I, Ramadan K, Stivala LA, Vannini V, Hübscher U, Spadari S, and Maga G (2005) Inhibition of mammalian DNA polymerases by resveratrol: mechanism and structural determinants. Biochem $J \mathbf{3 8 9}$ : $259-268$.

Longley DB and Johnston PG (2005) Molecular mechanisms of drug resistance. $J$ Pathol 205:275-292.

Lu C-H, Engelmann NJ, Lila MA, and Erdman JW, Jr (2008) Optimization of lycopene extraction from tomato cell suspension culture by response surface methodology. J Agric Food Chem 56:7710-7714.

Ma X, Tian X, Huang X, Yan F, and Qiao D (2007) Resveratrol-induced mitochondrial dysfunction and apoptosis are associated with $\mathrm{Ca} 2+$ and mCICR-mediated MPT activation in HepG2 cells. Mol Cell Biochem 302:99-109.

Marusyk A and Polyak K (2010) Tumor heterogeneity: causes and consequences. Biochim Biophys Acta 1805:105-117.

Miki H, Uehara N, Kimura A, Sasaki T, Yuri T, Yoshizawa K, and Tsubura A (2012) Resveratrol induces apoptosis via ROS-triggered autophagy in human colon cancer cells. Int J Oncol 40:1020-1028.

Oprea-Lager DE, Bijnsdorp IV, VAN Moorselaar RJ, VAN DEN Eertwegh AJ, Hoekstra OS, and Geldof AA (2013) ABCC4 decreases docetaxel and not cabazitaxel efficacy in prostate cancer cells in vitro. Anticancer Res 33:387-391.

Paller CJ and Antonarakis ES (2011) Cabazitaxel: a novel second-line treatment for metastatic castration-resistant prostate cancer. Drug Des Devel Ther 5:117-124.

Pan L, Chai H-B, and Kinghorn AD (2012) Discovery of new anticancer agents from higher plants. Front Biosci (Schol Ed) 4:142-156. 
Patel P, Ashdown D, and James N (2004) Is gene therapy the answer for prostate cancer? Prostate Cancer Prostatic Dis 7 (Suppl 1):S14-S19.

Peterson JA, Oblad RV, Mecham JC, and Kenealey JD (2016) Resveratrol inhibits plasma membrane $\mathrm{Ca}^{2+}$-ATPase inducing an increase in cytoplasmic calcium. Biochem Biophys Rep 7:253-258.

Petrylak DP, Tangen CM, Hussain MH, Lara PN Jr, Jones JA, Taplin ME, Burch PA Berry D, Moinpour C, Kohli M, et al. (2004) Docetaxel and estramustine compared with mitoxantrone and prednisone for advanced refractory prostate cancer. $N$ Engl J Med 351:1513-1520.

Pinthus JH, Waks T, Malina V, Kaufman-Francis K, Harmelin A, Aizenberg I, Kanety H, Ramon J, and Eshhar Z (2004) Adoptive immunotherapy of prostate cancer bone lesions using redirected effector lymphocytes. J Clin Invest 114:1774-1781.

Shao X, Gao D, Wang Y, Jin F, Wu Q, and Liu H (2016) Application of metabolomics to investigate the antitumor mechanism of flavopiridol in MCF-7 breast cancer cells. J Chromatogr B Analyt Technol Biomed Life Sci 1025:40-47.

Siegel RL, Miller KD, and Jemal A (2018) Cancer statistics, 2018. CA Cancer J Clin 68:7-30.

Singh A and Settleman J (2010) EMT, cancer stem cells and drug resistance: an emerging axis of evil in the war on cancer. Oncogene 29:4741-4751.

Tannock IF, de Wit R, Berry WR, Horti J, Pluzanska A, Chi KN, Oudard S, Théodore C, James ND, Turesson I, et al.; TAX 327 Investigators (2004) Docetaxel plus prednisone or mitoxantrone plus prednisone for advanced prostate cancer. $N$ Engl $J$ Med 351:1502-1512.

Theyer G, Schirmböck M, Thalhammer T, Sherwood ER, Baumgartner G, and Hamilton G (1993) Role of the MDR-1-encoded multiple drug resistance phenotype in prostate cancer cell lines. J Urol 150:1544-1547.

Tilley WD, Bentel JM, Aspinall JO, Hall RE, and Horsfall DJ (1995) Evidence for a novel mechanism of androgen resistance in the human prostate cancer cell line, PC-3. Steroids 60:180-186.

Zhao A, Chen F, Ning C, Wu H, Song H, Wu Y, Chen R, Zhou K, Xu X, Lu Y, et al. (2017) Use of real-time cellular analysis and Plackett-Burman design to develop the serum-free media for PC-3 prostate cancer cells. PLoS One 12: e0185470.

Zimmermann GR, Lehár J, and Keith CT (2007) Multi-target therapeutics: when the whole is greater than the sum of the parts. Drug Discov Today 12:34-42.

Address correspondence to: Jason Kenealey, Department of Nutrition, Dietetics and Food Science, Brigham Young University, S-221 ESC, Provo, UT 84602. E-mail: jason_kenealey@byu.edu 